\section{Normalización escala Early Feeding Skills (EFS) en recién nacidos prematuros}

\section{Standardization of Early Feeding Skills (EFS) scale in preterm infants}

\author{
Abarzúa P. Constanza ${ }^{\mathrm{a}}$, Godoy M. Ana ${ }^{\mathrm{b}, \mathrm{c}}$, Rubilar P. Mariana ${ }^{\mathrm{f}}$, \\ Silva Sch. María ${ }^{\mathrm{b}, \mathrm{c}}$, Velásquez Z. Mónica ${ }^{\mathrm{b}, \mathrm{c}, \mathrm{d}}$, Bustos M. Luis ${ }^{\mathrm{d}, \mathrm{e}}$
}

\author{
aDepartamento de Administración de Educación Municipal, Ilustre Municipalidad de Vilcún, Chile \\ ${ }^{\text {b} C}$ arrera de Fonoaudiología, Universidad de La Frontera, Temuco, Chile \\ 'Departamento de Especialidades Médicas, Universidad de la Frontera, Temuco, Chile \\ dDepartamento Salud Pública, Universidad de la Frontera, Temuco, Chile \\ 'Centro de Excelencia CIGES, Universidad de la Frontera, Temuco, Chile \\ fDepartamento de Administración de Educación Municipal, Ilustre Municipalidad de Loncoche, Chile
}

Recibido: 28 de enero de 2019; aceptado: 7 de mayo de 2019

\section{Resumen}

La escala Early Feeding Skills (EFS) evalúa la conducta del neonato antes, durante y posterior al proceso de alimentación. Objetivos: Determinar la validez de fachada, de contenido y evaluar con dicha escala el proceso de alimentación en los recién nacidos prematuros comparando con variables relevantes. Pacientes y Método: Se evaluaron prematuros de 34 a 36 semanas de edad corregida sin daño neurológico o malformaciones craneofaciales. Diseño de tipo descriptivo de corte transversal. Los datos fueron obtenidos mediante escala EFS, versión corregida mediante validación de fachada y contenido. Las variables de estudio fueron género, logro de alimentación por pecho, y habilidades de alimentación durante el proceso de alimentación, evaluadas por 2 observadoras Se analizaron medidas de dispersión y se aplicó prueba Fisher al 5\% de significancia, estableciendo la asociación de los resultados obtenidos con las variables. Resultados: Se evaluaron 5 dominios: (1) Capacidad de mantenerse enfocado en la alimentación, en que el 75,3\% posee un nivel desempeño deficiente y el $28,6 \%$ equitativo existiendo diferencias significativas con la variable sexo. (2) Capacidad de organizar el funcionamiento motor - oral tiene un $10,5 \%$ deficiente, un $68,8 \%$ equitativo y un $20,6 \%$ bueno. (3) Capacidad de coordinar la deglución el $95,2 \%$ presenta un rendimiento equitativo. (4) Capacidad de mantener la estabilidad fisiológica el 96,7\% fue equitativo y en (5) Evaluación de la tolerancia alimentaria oral tiene un desempeño deficiente del 41,6\%. Conclusión: La escala EFS es una herramienta que aporta información relevante para describir el proceso de alimentación oral en lactantes prematuros, permitiendo identificar las áreas de mayor dificultad que requieren tratamiento profesional, sin embargo, esta herramienta no es suficiente por sí sola para llevar a cabo una evaluación integral del proceso de alimentación del neonato.
Palabras clave:

Prematuro; alimentación oral; evaluación; deglución 


\begin{abstract}
Introduction: The Early Feeding Skills (EFS) scale assessed the neonate's behavior before, during, and after the feeding process. Objectives: To determine the face and content validity, and to evaluate with this scale the feeding process in premature newborns comparing with relevant variables. Patients and Method: Premature newborns were evaluated; they were between 34 to 36 weeks of corrected age, without neurological damage or craniofacial malformations. Cross-sectional descriptive study. The data were obtained through the EFS scale, version corrected by face and content validation. The study variables were gender, breastfeeding achievement, and feeding skills during the feeding process, evaluated by two observers. Dispersion measures were analyzed, and the Fisher test was used at 5\% significance, establishing the association of the obtained results with the variables. Results: 5 domains were evaluated: (1) Ability to stay focused on food, in which $75.3 \%$ have a poor performance level, and $28.6 \%$ equitable, with significant differences in the sex variable. (2) Ability to organize motororal functioning presents $10.5 \%$ deficient, $68.8 \%$ equitable, and $20.6 \%$ good. (3) Ability to coordinate swallowing $95.2 \%$ presents an equitable performance. (4) Ability to maintain physiological stability $96.7 \%$ was equitable; and (5) Evaluation of oral food tolerance presents $41.6 \%$ of poor performance. Conclusion: The EFS scale is a tool that provides relevant information to describe the oral feeding process in premature infants, allowing to identify the areas of greatest difficulty that require professional treatment, however, this tool is not enough by itself to carry a comprehensive evaluation of the newborn feeding process.
\end{abstract}

Keywords:

Preterm; oral feeding; swallowing; evaluation

\section{Introducción}

En los recién nacidos la alimentación oral es un proceso complejo, que para llegar a ser exitoso depende de la indemnidad anatómo-funcional de las estructuras orofaciales que favorecen el proceso succión deglución, las que se ven afectadas de sobremanera en los casos de prematurez ${ }^{1-3}$.

Un neonato es considerado prematuro al exponerse a la vida extrauterina antes de las 37 semanas, lo que conlleva a diversas comorbilidades que se manifiestan en: deficiente control postural, control motor oral disminuido, inmadurez gastrointestinal y dificultad en el control fisiológico; estas dificultades interfieren la alimentación oral segura, efectiva, competente y confortable $e^{4,5}$. Es importante la existencia de pautas de evaluación y seguimiento del proceso de alimentación en los bebés prematuros, que faciliten el trabajo del equipo de la Unidad de Paciente Critico Neonatal ${ }^{6,7}$. Diversos autores plantean escalas como el Instrumento de Evaluación de la Prontitud de los Prematuros para Iniciación de la Alimentación por Vía Oral ${ }^{8,9}$ y la Escala de Evaluación Motor Oral Neonatal ${ }^{10}$, las cuales plantean un predictor de funciones motoras orales para la alimentación por boca en lactantes ${ }^{8,10,11}$. En el presente documento se analiza la escala Early Feeding Skills (EFS), la cual evalúa la conducta del neonato antes, durante y posterior al proceso de alimentación, además permite identificar áreas descendidas y determinar lineamientos para recibir posteriormente un tratamiento fonoaudiológico acorde a las necesidades y habilidades presentes en el neonato ${ }^{5,12,13}$. El objetivo fue describir a través de la escala EFS el proceso de alimentación de recién nacidos prematuros. Objetivos específicos fueron: 1) Determinar la validez de fachada de la escala EFS; 2) Determinar la validez de contenido de la escala EFS; 3 ) Evaluar el proceso de alimentación con escala de observación EFS, y 4) Analizar los resultados de los ítems para cada uno de los dominios según variables de estudio y nivel de desempeño o puntuación obtenida en cada dominio (adecuado, equitativo o deficiente).

\section{Pacientes y Método}

Se incluyeron 138 neonatos prematuros, periodo 2015 - 2016 de la Unidad de Neonatología del Hospital Dr. Hernán Henríquez Aravena de la ciudad de Temuco, Chile, edad gestacional corregida 34 a 36 semanas, excluyendo a aquellos que presentan malformaciones craneofaciales y enfermedades neurológicas. Los datos fueron obtenidos mediante escala EFS, versión corregida mediante validación de fachada y contenido.

\section{Validez del instrumento}

Se solicitó manual y puntuaciones de la escala a la autora Suzanne Thoyre, luego se realizó validez de fachada mediante la traducción del instrumento de inglés a español por un hablante nativo del inglés y posteriormente se efectuó un análisis del español al inglés, comprobando que el instrumento no perdiera coherencia $^{12}$.

Una vez traducido el instrumento al español, fue sometido a evaluación por 6 fonoaudiólogos expertos en el área, dichos profesionales responden a una pauta que considera aspectos de suficiencia, claridad, cohe- 
rencia y relevancia, asignando una puntuación de 1 a 4, considerando los valores 3 y 4 (puntuación moderada y alta) como óptimas para la validación de cada aspecto, para los ítems que fueron evaluados con 1 y 2 (puntuación baja y muy baja) por al menos dos de los expertos, se modificaron a través de sugerencias realizadas por los mismos. Finalmente se dieron a conocer las modificaciones de ítems a todos los fonoaudiólogos expertos, quienes concordaron en todas las áreas evaluadas con puntuación alta (gráfico 1). Posteriormente se evaluó el grado de concordancia entre los expertos, estimando que cada ítem debía ponderar al menos el 67\% del total de las respuestas. Luego se analizaron grupos de ítems, encontrándose en cada grupo la descripción de distintos procesos y momentos de la alimentación del neonato tales como: la capacidad de mantenerse enfocado en la alimentación, la organización motora oral, la deglución, la estabilidad fisiológica y la tolerancia a la alimentación, dichos grupos son denominados dominios. Posteriormente se realizaron cruces entre los dominios y las variables descritas en la metodología.

El diseño de la investigación correspondió a un estudio de corte transversal. Se analizaron los datos obtenidos de la evaluación con escala EFS mediante medidas de dispersión (mediana, media y promedio). Se aplicó prueba Fisher al 5\% de significancia, estableciendo la asociación de los resultados agrupados en dominios con las variables seleccionadas. Las variables de estudio y puntaje asignado a cada una de las opciones fueron:

1. Sexo del paciente:

a. Hombre: 1 (Se considera valor menor, debido a que el hombre presenta mayor probabilidad a presentar dificultades de alimentación al nacer). b. Mujer: 2 .
2. Logro de alimentación por pecho.
a. No: 1 .
b. Sí: 2 .

3. Habilidades de alimentación durante el proceso de alimentación.
a. Disminuyen: 1.
b. Se mantienen: 2 .
c. Aumentan: 3 .

La observación fue realizada por dos evaluadoras en paralelo, las que posteriormente consensuaron las puntuaciones asignadas en cada ítem. Las evaluaciones se realizaron durante los periodos de alimentación establecidos por el servicio. La leche utilizada es de preferencia materna y como segunda opción fórmula, en la medida indicada por el médico. Durante el proceso de alimentación la madre debía estar presente, siendo ella quien recibe la explicación en detalle del procedimiento, dando lectura al consentimiento informado correspondiente a la observación. En el transcurso de la alimentación se brinda apoyo, guiando a la madre y entregando las sugerencias pertinentes respecto a la postura, pausas necesarias y agarre adecuado del pecho materno. Además, durante el proceso se monitorean los signos vitales, los que al variar sobrepasando rangos de normalidad, constituyen motivo suficiente para interrumpir o finalizar el proceso, completando la ingesta necesaria de alimento por sonda.

El trabajo de investigación fue aprobado por el Comité de Ética del Servicio de Salud Araucanía Sur, que fue presentado al Director del Hospital Dr. H.H.A. obteniendo los permisos pertinentes para realizar las evaluaciones en el Servicio de Neonatología.
Gráfico 1. Puntuación asignada por cada evaluador en cada aspecto. Se considera el promedio del puntaje obtenido en los ítems

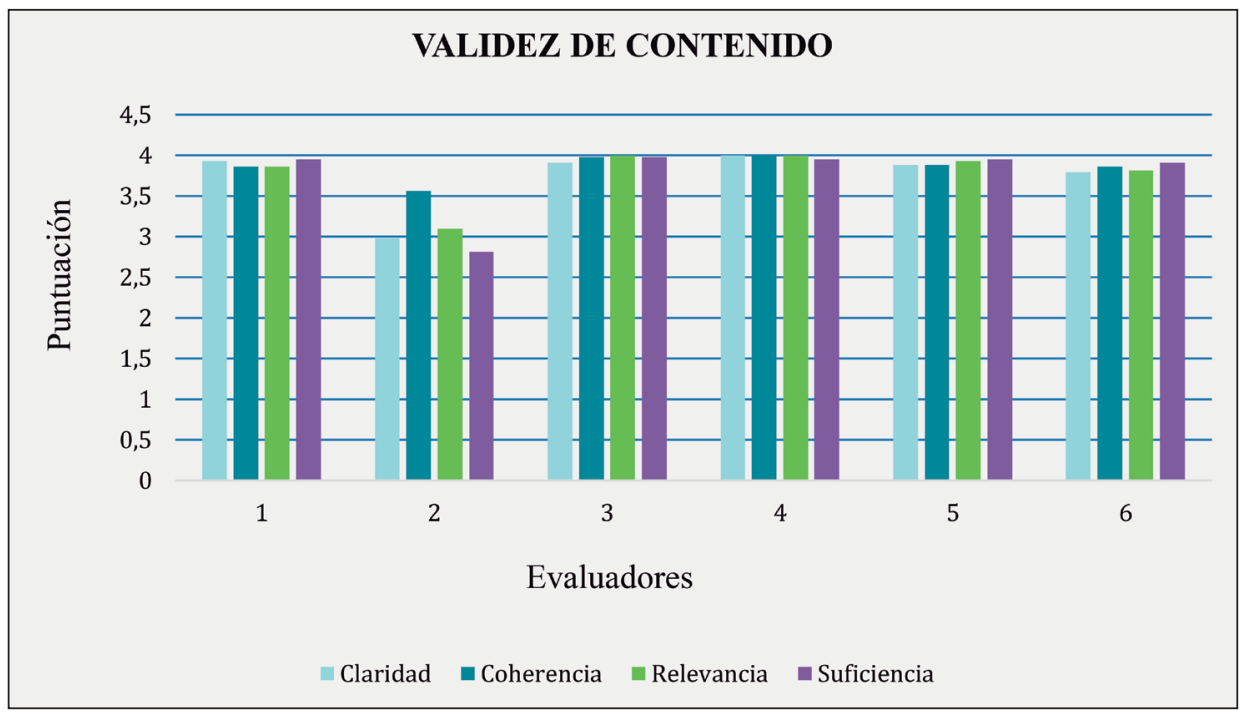




\section{Resultados}

De los recién nacidos evaluados el 50,7\% correspondió a mujeres y el $49,3 \%$ a hombres, los que fueron observados por dos evaluadoras, obteniendo un total de 276 mediciones. La Capacidad de mantenerse enfocado en la alimentación, con la variable sexo mostró que el nivel de desempeño deficiente era mayor en los hombres, y el nivel equitativo era más alto en mujeres, siendo estas diferencias significativas. El nivel de desempeño en la Capacidad de organizar el funcionamiento motor oral no evidencio diferencias significativas con la variable sexo. En cuanto a la Capacidad de coordinar la deglución presentó un rendimiento mayormente equitativo, así como en el dominio de la Capacidad de mantener la estabilidad fisiológica, entre estos dominios y la variable sexo no se encontraron diferencias significativas. Con respecto a la Evaluación de la tolerancia alimentaria oral y el sexo, no se encontró una asociación (Tabla 1).

Los cinco dominios estudiados se confrontaron con la variable Logra alimentarse por pecho, evidenciando diferencias significativas en los niveles de desempeño. Se destaca que en la Capacidad de mantenerse enfocado en la alimentación y en la Evaluación de la tolerancia alimentaria oral, los neonatos presentan porcentajes altos en el nivel de desempeño deficiente (Tabla 2).

Tabla 1. Comparación de los niveles de desempeño de la escala EFS según sexo

\begin{tabular}{|c|c|c|c|c|c|}
\hline \multirow[t]{2}{*}{ Dominio } & \multirow[t]{2}{*}{ Sexo } & \multicolumn{4}{|c|}{ Niveles de desempeño } \\
\hline & & Deficiente & Equitativo & Bueno & $\mathrm{P}$ \\
\hline $\begin{array}{l}\text { Capacidad de mantenerse enfocado } \\
\text { en la alimentación }\end{array}$ & $\begin{array}{l}\text { Mujer } \\
\text { Hombre } \\
\text { Total }\end{array}$ & $\begin{array}{c}92(65,7) \\
116(85,3) \\
208(75,4)\end{array}$ & $\begin{array}{l}48(34,3) \\
20(14,7) \\
68(24,6)\end{array}$ & $\begin{array}{l}- \\
-\end{array}$ & $<0,001$ \\
\hline $\begin{array}{l}\text { Capacidad de organizar el } \\
\text { funcionamiento motor oral }\end{array}$ & $\begin{array}{l}\text { Mujer } \\
\text { Hombre } \\
\text { Total }\end{array}$ & $\begin{array}{c}13(9,3) \\
16(11,8) \\
29(10,5)\end{array}$ & $\begin{array}{c}97(69,3) \\
93(68,4) \\
190(68,8)\end{array}$ & $\begin{array}{l}30(21,4) \\
27(19,8) \\
57(20,7)\end{array}$ & 0,771 \\
\hline Capacidad de coordinar la deglución & $\begin{array}{l}\text { Mujer } \\
\text { Hombre } \\
\text { Total }\end{array}$ & $\begin{array}{c}6(6,4) \\
4(2,9) \\
13(4,7)\end{array}$ & $\begin{array}{l}131(93,6) \\
132(97,1) \\
263(95,3)\end{array}$ & $\begin{array}{l}- \\
- \\
-\end{array}$ & 0,256 \\
\hline $\begin{array}{l}\text { Capacidad de mantener la estabilidad } \\
\text { fisiológica }\end{array}$ & $\begin{array}{l}\text { Mujer } \\
\text { Hombre } \\
\text { Total }\end{array}$ & $\begin{array}{l}2(1,4) \\
7(5,1) \\
9(3,3)\end{array}$ & $\begin{array}{l}138(98,6) \\
129(94,9) \\
267(96,7)\end{array}$ & $\begin{array}{l}- \\
- \\
-\end{array}$ & 0,099 \\
\hline $\begin{array}{l}\text { Evaluación de la tolerancia alimentaria } \\
\text { oral }\end{array}$ & $\begin{array}{l}\text { Mujer } \\
\text { Hombre } \\
\text { Total }\end{array}$ & $\begin{array}{l}49(35,0) \\
66(48,5) \\
115(41,7)\end{array}$ & $\begin{array}{l}57(40,7) \\
46(33,8) \\
103(37,3)\end{array}$ & $\begin{array}{l}24(24,3) \\
24(17,7) \\
58(21,0)\end{array}$ & 0,069 \\
\hline
\end{tabular}

Datos expresados en frecuencias y (\%). Prueba Exacta de Fisher.

Tabla 2. Comparación de los niveles de desempeño de la escala EFS según logra alimentarse por pecho

\begin{tabular}{|c|c|c|c|c|c|}
\hline \multirow[t]{2}{*}{ Dominio } & \multirow{2}{*}{$\begin{array}{l}\text { Logra alimentarse } \\
\text { por pecho }\end{array}$} & \multicolumn{4}{|c|}{ Niveles de desempeño } \\
\hline & & Deficiente & Equitativo & Bueno & $\mathrm{P}$ \\
\hline $\begin{array}{l}\text { Capacidad de mantenerse enfocado en la } \\
\text { alimentación }\end{array}$ & $\begin{array}{c}\text { No } \\
\text { Si } \\
\text { Total }\end{array}$ & $\begin{array}{c}16(61,5) \\
192(76,8) \\
208(75,4)\end{array}$ & $\begin{array}{l}10(38,5) \\
58(23,2) \\
68(24,6)\end{array}$ & $\begin{array}{l}- \\
- \\
-\end{array}$ & 0,097 \\
\hline $\begin{array}{l}\text { Capacidad de organizar el funcionamiento } \\
\text { motor oral }\end{array}$ & $\begin{array}{c}\text { No } \\
\text { Si } \\
\text { Total }\end{array}$ & $\begin{array}{c}2(7,7) \\
27(10,8) \\
29(10,5)\end{array}$ & $\begin{array}{c}18(69,2) \\
172(68,8) \\
190(68,8)\end{array}$ & $\begin{array}{c}6(23,1) \\
51(20,4) \\
57(20,7)\end{array}$ & 0,898 \\
\hline Capacidad de coordinar la deglución & $\begin{array}{c}\text { No } \\
\text { Si } \\
\text { Total }\end{array}$ & $\begin{array}{c}2(7,7) \\
11(4,4) \\
13(4,7)\end{array}$ & $\begin{array}{c}24(92,3) \\
239(95,6) \\
263(95,3)\end{array}$ & $\begin{array}{l}- \\
- \\
-\end{array}$ & 0,351 \\
\hline $\begin{array}{l}\text { Capacidad de mantener la estabilidad } \\
\text { fisiológica }\end{array}$ & $\begin{array}{c}\text { No } \\
\text { Si } \\
\text { Total }\end{array}$ & $\begin{array}{l}2(7,6) \\
7(2,8) \\
9(3,3)\end{array}$ & $\begin{array}{c}24(92,3) \\
243(97,2) \\
267(96,7)\end{array}$ & $\begin{array}{l}- \\
- \\
-\end{array}$ & 0,204 \\
\hline $\begin{array}{l}\text { Evaluación de la tolerancia alimentaria } \\
\text { oral }\end{array}$ & $\begin{array}{l}\text { No } \\
\text { Si } \\
\text { Total }\end{array}$ & $\begin{array}{l}10(38,5) \\
105(42,0) \\
115(41,7)\end{array}$ & $\begin{array}{c}10(38,5) \\
93(37,2) \\
103(37,3)\end{array}$ & $\begin{array}{c}6(23,0) \\
52(20,8) \\
58(21,0)\end{array}$ & 0,930 \\
\hline
\end{tabular}

Datos expresados en frecuencias y (\%). Prueba Exacta de Fisher. 
Tabla 3. Comparación de los niveles de desempeño de la escala EFS según habilidades durante la alimentación

\begin{tabular}{|c|c|c|c|c|c|}
\hline \multirow[t]{2}{*}{ Dominio } & \multirow{2}{*}{$\begin{array}{l}\text { Habilidades durante } \\
\text { la alimentación }\end{array}$} & \multicolumn{4}{|c|}{ Niveles de desempeño } \\
\hline & & Deficiente & Equitativo & Bueno & $P$ \\
\hline $\begin{array}{l}\text { Capacidad de mantenerse } \\
\text { enfocado en la alimentación }\end{array}$ & $\begin{array}{l}\text { Disminuye } \\
\text { Mantiene } \\
\text { Mejora } \\
\text { Total }\end{array}$ & $\begin{array}{c}112(92,6) \\
75(57,7) \\
21(8) \\
208(75,4)\end{array}$ & $\begin{array}{c}9(7,4) \\
55(42,3) \\
4(16) \\
68(24,6)\end{array}$ & $\begin{array}{l}- \\
- \\
- \\
-\end{array}$ & $<0,000$ \\
\hline $\begin{array}{l}\text { Capacidad de organizar el } \\
\text { funcionamiento motor oral }\end{array}$ & $\begin{array}{l}\text { Disminuye } \\
\text { Mantiene } \\
\text { Mejora } \\
\text { Total }\end{array}$ & $\begin{array}{c}24(19,8) \\
4(3,1) \\
1(4,0) \\
29(10,5)\end{array}$ & $\begin{array}{c}90(74,4) \\
77(59,2) \\
23(92,0) \\
190(68,8)\end{array}$ & $\begin{array}{c}7(5,8) \\
49(37,7) \\
1(4,0) \\
57(20,7)\end{array}$ & $<0,000$ \\
\hline $\begin{array}{l}\text { Capacidad de coordinar la } \\
\text { deglución }\end{array}$ & $\begin{array}{l}\text { Disminuye } \\
\text { Mantiene } \\
\text { Mejora } \\
\text { Total }\end{array}$ & $\begin{array}{c}9(7,4) \\
2(1,5) \\
2(8,0) \\
13(4,7)\end{array}$ & $\begin{array}{c}112(92,6) \\
128(98,5) \\
23(92,0) \\
273(95,3)\end{array}$ & $\begin{array}{l}- \\
- \\
- \\
-\end{array}$ & 0,040 \\
\hline $\begin{array}{l}\text { Capacidad de mantener la } \\
\text { estabilidad fisiológica }\end{array}$ & $\begin{array}{l}\text { Disminuye } \\
\text { Mantiene } \\
\text { Mejora } \\
\text { Total }\end{array}$ & $\begin{array}{c}7(5,8) \\
2(1,5) \\
- \\
9(3,3)\end{array}$ & $\begin{array}{l}114(94,2) \\
128(98,5) \\
25(100,0) \\
267(96,7)\end{array}$ & $\begin{array}{l}- \\
- \\
- \\
-\end{array}$ & 0,144 \\
\hline $\begin{array}{l}\text { Evaluación de la tolerancia } \\
\text { alimentaria oral }\end{array}$ & $\begin{array}{l}\text { Disminuye } \\
\text { Mantiene } \\
\text { Mejora } \\
\text { Total }\end{array}$ & $\begin{array}{c}75(62,0) \\
32(24,6) \\
8(32,0) \\
115(41,7)\end{array}$ & $\begin{array}{c}36(29,7) \\
55(42,3) \\
12(48,0) \\
103(37,3)\end{array}$ & $\begin{array}{c}10(8,3) \\
43(33,1) \\
5(20,0) \\
58(21,0)\end{array}$ & $<0,000$ \\
\hline
\end{tabular}

Datos expresados en frecuencias y (\%). Prueba Exacta de Fisher.

El dominio de Capacidad de mantener la estabilidad fisiológica no se asoció con las Habilidades durante la alimentación. Se observaron diferencias importantes en los niveles de desempeño del dominio Capacidad de mantenerse enfocado en la alimentación en cada categoría de las Habilidades durante la alimentación, siendo mayor el nivel deficiente en aquellos neonatos que presentaron una disminución de las Habilidades durante la alimentación. En cuanto a la Capacidad de organizar el funcionamiento motor oral los porcentajes del nivel deficiente fueron más bajos que los equitativo y bueno, siendo estas asociaciones significativas. En el dominio de Capacidad para coordinar la deglución se asoció significativamente con las Habilidades durante la alimentación, observándose una distribución del nivel deficiente menor al equitativo. En la Evaluación de la tolerancia alimentaria oral se observaron diferencias significativas en los tres niveles de desempeño respecto de las Habilidades durante la alimentación (Tabla 3).

\section{Discusión}

Del estudio realizado se observa que la escala EFS es un predictor de las habilidades de alimentación en los prematuros estudiados. El cruce de los dominios con las variables, en función al desempeño de los neonatos durante el proceso de alimentación, indica que presentan mayor porcentaje de desempeño equitativo en los dominios Capacidad de organizar el funcionamiento motor oral y Capacidad de coordinar la deglución; esto permite que ellos puedan iniciar la alimentación por vía oral a través del pecho materno. Sin embargo, esto no significa que puedan recibir todo el alimento necesario por vía oral, en ocasiones requiere ser completada su nutrición a través de una vía alternativa de alimentación como la sonda nasogástrica u orogástrica. Dicha situación se ve reflejada en los dominios Capacidad de mantenerse enfocado en la alimentación y Evaluación de la tolerancia alimentaria oral, los cuales tienen un mayor desempeño deficiente. Por otra parte, cabe destacar que el dominio Capacidad de mantener la estabilidad fisiológica tiene un mayor desempeño equitativo, el equipo investigador relaciona este resultado a que el neonato se alimentó al pecho materno, manteniéndose contenido por su madre y durante un tiempo limitado, ya que su nivel de energía no permite que se mantenga más minutos recibiendo alimento por vía oral. Thoyre et al el 2005 describió la escala EFS, mencionando que es un medio para identificar áreas de fortaleza y áreas en las que requiere apoyo un bebé prematuro para lograr una alimentación segura y efectiva, encontrándose en concordancia con lo observado en este estudio ${ }^{12}$. Por otra parte, indica que los bebés experimentan con frecuencia episodios de desaturación de oxígeno, mayor gasto de energía en respuesta al estrés y presencia de fatiga, lo cual se aprecia en los datos mencionados anteriormente ${ }^{13}$.

La escala EFS ha sido analizada en otros estudios, en el año 2017 en Brasil, mediante el estudio "Vali- 
dación para la población portuguesa de la Escala de Observación de las Habilidades Tempranas en la Alimentación Oral" ${ }^{(14)}$, que considera una muestra de 698 recién nacidos de edad gestacional $\geq 24$ y $<37$ semanas, en ella se estudia la validez y la fiabilidad, concluyendo que es una escala sensible, válida y fiable para observar las competencias tempranas del RN durante la alimentación oral, permite elaborar un plan de intervención y realizar un trabajo en conjunto a las familias.

Otro estudio que destaca en el área es "Validación de una escala clínica de la succión nutricia"15 que consideró una muestra de 179 lactantes $<6$ meses de edad y 86 lactantes con alto riesgo de alteración de la succión, la cual presenta validación de fachada y validación psicométrica, establece relación entre el desempeño de succión y el volumen ingerido, la deglución con la saturación periférica y la ventilación con la frecuencia respiratoria. Con dicha escala se fijaron patrones de alteración según antecedentes de afección neurológica, estabilidad hemodinámica e inmadurez.

No obstante a lo descrito anteriormente, el equipo evaluador considera que la escala debe complementar la evaluación con la observación de habilidades en succión no nutritiva de forma objetiva, el apego con la madre y la anatomía del pecho, ya que éstos son aspectos que pueden afectar directamente el desempeño en la alimentación de los neonatos. En la literatura destaca el instrumento "Prontitud de alimentación oral en prematuros" de Fujinaga et al (9), el cual tiene validez de contenido en Brasil y posibilita una evaluación para iniciar la alimentación por vía oral del prematuro, calificando a través de puntuación la tonicidad orofacial, reflejos adaptativos, succión no nutritiva y signos de estrés ${ }^{(11)}$. Cada una de las escalas mencionadas puede complementarse para evaluar íntegramente al lactante, pero deben ser utilizadas por profesionales especializados y competentes en el área, que puedan observar mediante un lineamiento común todos los aspectos importantes del complejo proceso de alimentación en esta población.

\section{Conclusión}

La escala EFS es una herramienta que aporta información relevante para describir el proceso de alimentación oral en lactantes prematuros, permitiendo identificar las áreas de mayor dificultad que requieren tratamiento profesional, sin embargo, esta herramienta no es suficiente por sí sola para llevar a cabo una evaluación integral del proceso de alimentación del neonato, considerando importante realizar previamente una adecuada evaluación de un profesional fonoaudiólogo, que poniendo en uso los conocimientos del área, observe además aspectos anatómicos y funcionales de la cavidad oral, reflejos adaptativos y pueda calificar su desempeño en la succión no nutritiva antes de observar el rendimiento de la succión nutritiva a través de la escala EFS.

\section{Responsabilidades Éticas}

Protección de personas y animales: Los autores declaran que los procedimientos seguidos se conformaron a las normas éticas del comité de experimentación humana responsable y de acuerdo con la Asociación Médica Mundial y la Declaración de Helsinki.

Confidencialidad de los datos: Los autores declaran que han seguido los protocolos de su centro de trabajo sobre la publicación de datos de pacientes.

Derecho a la privacidad y consentimiento informado: Los autores han obtenido el consentimiento informado de los pacientes y/o sujetos referidos en el artículo. Este documento obra en poder del autor de correspondencia.

\section{Conflicto de intereses}

Los autores declaran no tener conflicto de intereses.

\section{Agradecimientos}

Agradecemos a Suzanne Thoyre, autora de EFS por facilitar la escala, manual y puntajes de la escala. A la Doctora María Angélica Belmar, jefa de unidad de Neonatología de Hospital Dr. HHA, que permitió y facilitó en conjunto al personal de la unidad, la aplicación de la escala. Y finalmente a los expertos que participaron como evaluadores, aportando con su conocimiento y experiencia en la validez de contenido. 


\section{Referencias}

1. Villanueva P, Palomino H.

Funciones orofaciales en el neonato. En: Motricidad Orofacial Fundamentos anatomofisiológicos y evolutivos para la evolución clínica. 1a. ed., Santiago: Editorial Universitaria 2011;59-64.

2. Durán A, Rodríguez MA, de la Teja E, et al. Succión, deglución, masticación y sentido del gusto prenatales. Desarrollo sensorial temprano de la boca. Acta Pediatr Mex. 2012;33:137-41.

3 American Speech-Language-Hearing Association. (2001). Roles of Speech Language Pathologists in Swallowing and Feeding Disorders: Technical Report [Technical Report]. Available from www. asha.org/policy.

4. Campos Z. Problemas de la alimentación en lactantes, Primera parte: generalidades. Acta pediátr costarric. 2009;21:18-25.

5. da Costa, SP, van den Engel-Hoek, Bos A. Sucking and swallowing in infants and dignostic tools. Journal of perinatology. 2008;28:247-57.

6. Durán A, Castillo E, de la Teja E, et al. Alimentación difícil en el paciente neonato, el enfoque estomatológico. Reporte de un caso. Revista Odontológica Mexicana. 2012;16:285-93.

7. Arvedson J, Brodsky L. Pediatric Swallowing and Feeding: Assessment and Management. 2a. ed., Canada: Editorial Singular Thomson Learning 2001;283340.

8. Fujinaga CI, Zamberlan NE, Rodarte MD, Scochi CG. Reliability of an instrument to assess the readiness of preterm infants for oral feeding. Pro Fono. 2007; 19:143-50.

9. Fujinaga C, Scochi C, Santos C et al. Validação do conteúdo de um instrumento para avaliação da prontidão do prematuro para início da alimentação oral. Rev. Bras. Saude Mater. Infant. 2008; 8: 391-9.

10. Da Costa SP, van der Schans CP. The reliability of the Neonatal Oral-Motor Assessment Scale. Acta Paediatr. 2008; 97:21-6.

11. La Orden E, Salcedo E, Cuadrado I, et al. Retraso de la adquisición de la succióndeglución-respiración en el pretérmino: efectos de una estimulación precoz. Nutrición Hospitalaria. 2012; 27:1120-6.

12. Thoyre S, Shaker C, Pridham K. The Early Feeding Skills Assessment for Preterm Infants. Neonatal Netw. 2005;24:7-16.

13. Ministerio De Salud. Guía Clínica: Prevención parto prematuro. Revisión y actualización. 2010;7-28.

14. Santos M, Maroco J, Vasconcellos T, et al. Validation of the Early Feeding Skills Assessment Scale for the Portuguese population. Revista de Enfermagem Referência. 2017;4:131-42.

15. Rendón M, Villasís M, Martínez M. Validación de una escala clínica de la succión nutricia. Rev Med Inst Mex Seguro Soc. 2016;54:318-26. 